\section{Reißt endlich die Mauern ein!}

\author{
Warum sind ambulanter und stationärer Sektor immer noch so radikal \\ getrennt? Hier sind drei Rezepte für eine bessere Patientenversorgung.
} scheitern - die Mauern zwischen dem ambulanten und dem stationären Sektor in der medizinischen Versorgung sind nur schwer zu Fall zu bringen. Dabei sind sich eigentlich alle Beteiligten einig, dass diese verkrustete Struktur endlich aufgebrochen werden muss. Erste Schritte hat der Gesetzgeber mit der ambulanten spezialfachärztlichen Versorgung (ASV) oder der Öffnung der Krankenhäuser für die ambulante Versorgung schon getan - wobei die ASV zeigt, wie es nicht funktionieren kann. Das Grundproblem bleibt indes ungelöst.

\section{Unter-, Über- und Fehlversorgung}

Dabei wäre es dafür allerhöchste Zeit. Die jetzigen Strukturen schaffen Unter-, Über- und Fehlversorgung. Die Versorgungslage ist von steigender Arztdichte und erheblichen Verteilungsproblemen geprägt. Im ländlichen Bereich fehlen mitunter Behandlungsmöglichkeiten in der Grundversorgung mit Haus- und Kinderärzten. Eine flächendeckende medizinische Versorgung der Bevölkerung wird damit zunehmend zur Herausforderung.

Auf der anderen Seite eine eklatante Überversorgung. Als Beispiel könnte, skizzenhaft dargestellt, die rheinländische Metropole Köln dienen. Hier gibt es bei den niedergelassenen fachärztlichen Internisten inklusive Kardiologen, einen Versorgungsgrad von $200 \%$. Zudem versorgen insgesamt 16 Kliniken die Patienten der Stadt und des
_ Gute Ideen versanden, Innovationen gibt es in Ballungszentren
Umlands im Bereich „Krankheiten und Störungen des Kreislaufsystems“. Diese Massierung von Versorgungsangeboten schafft eine Konkurrenzsituation niedergelassener Ärzte und zwischen den Kliniken. Sie birgt die Gefahr, dass medizinisch nicht notwendige Leistungen erbracht werden. Damit wird ggf. nicht nur das Geld der Versicherten verschwendet, es kann den Patienten sogar gesundheitlich schaden.

Längst reicht es also nicht mehr, nur an einigen Stellschrauben $\mathrm{zu}$ drehen. Gebraucht werden umfassendere Reformen der Versorgungslandschaft. Dafür sind drei Schritte notwendig.

Erstens sollte es künftig eine sektorenübergreifende Versorgung nach Versorgungsstufen geben. Die Hausärzte übernehmen weiterhin die Primärversorgung. Daneben gibt es eine allgemeine und eine spezialisierte fachärztliche Versorgung. Zu letzterer gehören etwa Humangenetiker und Laborärzte. Eine analoge Einteilung würde für den stationären Sektor gelten.

Die Kapazitäten in der Behandlung folgen dabei dem tatsächlichen Leis-

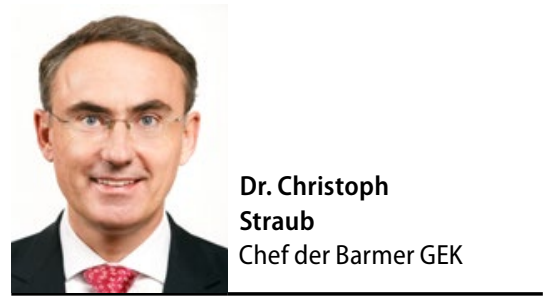

tungsbedarf, wodurch sich Überversorgung abbauen ließe. Erarbeitet würde die konkrete Planung durch ein sektorenübergreifendes Planungsgremium, an dem sich auch die Bundesländer beteiligen sollten.

Zweitens sollte das Prinzip "gleiche Leistung, gleiches Geld“ gelten. Gerade hier befindet sich das System in einer Schieflage. Ein Arzt sollte in seiner Praxis für die gleiche Leistung wie ein Krankenhaus vergütet werden. Ansonsten bestehen automatisch Fehlanreize. Denn es entspricht der Logik wirtschaftlichen Handelns, eine Leistung dort zu erbringen, wo sie mit einer höheren Vergütung belohnt wird.

\section{Wir brauchen regionale Verbünde}

Drittens braucht es regionale Versorgungsverbünde. Damit kann zweierlei gelingen: Zum einen wird die medizinische Versorgung im ländlichen Raum sichergestellt. Im urbanen Raum hingegen sollte auf Spezialisierung gesetzt werden. Schließlich gilt es, hier mehr Effizienz in der Versorgung zu schaffen und Doppelstrukturen abzubauen.

Diese Vorschläge werden wieder auf viele Widerstände stoßen. Allerdings macht Hoffnung, dass die schieren Fakten Zweifler zur Einsicht drängen. Schließlich drohen immer größere Verteilungsprobleme bei $\mathrm{zu}$ gleich steigender Arztdichte. Vielleicht wächst angesichts dieses Konfliktes die Bereitschaft, vermehrt zusammenzuarbeiten.

- Der Autor ist Vorstandsvorsitzender der Barmer GEK. 